\title{
Understanding the association between informed choice and long-term reversible contraception among women aged 15-49 in Uganda: findings from the 2016 Demographic Health Survey
}

Hallie Dau ( $\nabla$ hallie.dau@cw.bc.ca )

The University of British Columbia School of Population and Public Health https://orcid.org/00000001-6193-4032

Othman Kakaire

Makerere University Faculty of Medicine: Makerere University College of Health Sciences

Mohammad Ehsanul Karim

The University of British Columbia School of Population and Public Health

Carolyn Nakisige

Uganda Cancer Institute

Marianne Vidler

The University of British Columbia Faculty of Medicine

Beth A Payne

The University of British Columbia School of Population and Public Health

Gina Ogilvie

The University of British Columbia School of Population and Public Health

\section{Research Article}

Keywords: Uganda, contraception, informed choice, long-acting reversible contraception

Posted Date: March 8th, 2022

DOI: https://doi.org/10.21203/rs.3.rs-1356259/v1

License: (c) (1) This work is licensed under a Creative Commons Attribution 4.0 International License. Read Full License 


\section{Abstract}

\section{Introduction}

Long-acting reversible contraceptive (LARC) methods are superior to other modern birth control methods in preventing pregnancy as they are highly effective and have a low failure rate. The Ugandan Ministry of Health has prioritized providing long-acting reversible contraception (LARC) and ensuring informed choice within the context of family planning. This study aims to understand the relationship between informed choice and LARC use among women aged 15-49 in Uganda after adjusting for potential confounding.

\section{Methods}

This cross-sectional study utilizes data from the 2016 Uganda Standard Demographic and Health Survey. Thomas-Rao corrections to a chi-square test were used for the bivariable analysis. A design-adjusted multivariable logistic regression was used to estimate the association between informed choice and LARC use. Propensity score matching was conducted as a sensitivity analysis.

\section{Results}

In all, 3,646 women were included in the analysis and 975 reported using a LARC. In the design- adjusted multivariable analysis, the odds of reporting LARC usage were $1.98(95 \% \mathrm{Cl} 1.61-2.43)$ times higher among women who reported informed choice compared to those who did not. The subsequent propensity score analysis reported similar findings.

\section{Discussion}

Providing informed choices can help increase the number of women who utilize LARCs in Uganda. As such, the Ugandan Ministry of Health should further expand access to family planning counselling as it could contribute to the reduction of unplanned pregnancies across Uganda through the use of LARCs.

Significance: There is currently no research on the association between informed choice and long-term reversible contraception (LARC) use in Uganda. More broadly, research in relation to informed choice and LARC use in sub-Saharan Africa is largely descriptive. This association is important to understand because Uganda has one of the highest unplanned pregnancy and fertility rates in the world. The study will contribute to a better understanding of family planning counselling in Uganda, which may be useful in developing future family planning programs and interventions. As such, this will help reduce the overall unplanned pregnancy rate in Uganda.

\section{Introduction}

Long-acting reversible contraceptive (LARC) methods are superior to other modern birth control methods in preventing pregnancy as they are highly effective and have a low failure rate. $(1,2)$ LARCs include both 
the intrauterine device (IUD) and implant. Although short-acting reversible contraceptive methods such as the injectable and the pill remain the most popular type of contraception among women in sub-Saharan Africa, there has been a growing number of women opting for LARCs in recent years. $(3,4)$ In Uganda the number of women aged 15-49 using a LARC more than doubled from 2011 to 2016 from 3.2\% to $7.8 \%$.

(3) The number of users in Uganda is expected to continue to grow as demand increases for highly effective reversible contraceptive methods to limit childbearing.(5)

There is currently no research on the association between informed choice and LARC use in Uganda. Informed choice, within the context of contraception, is defined as a woman's ability to independently choose a contraceptive method based on knowledge of all available information.(6) This includes, but is not limited to, side effects, effectiveness, cost, and availability. Although there is similar research in neighboring sub-Saharan African countries, the majority of the results focus on the association of informed choice and general contraceptive use.(7-9) Furthermore, current published research in relation to informed choice and LARC usage in sub-Saharan Africa is largely descriptive.(10-13)

It is important to better understand the association between informed choice and LARC use in Uganda in order to address the high fertility (5.4 per women) and unplanned pregnancy $(41 \%)$ rates, which remain some of the highest in the world.(3) Furthermore, women in Uganda do not have access to abortion except in extenuating circumstances.(14) Providing highly effective birth control methods is all the more essential where safe abortion services are absent. Prior research has shown that women who are more informed about family planning choices are more likely to choose a highly effective LARC method.(1517) Furthermore, the Ugandan Ministry of Health has prioritized in the 2020-2025 National Family Planning Advocacy Strategy Costed Implementation Plan Ugandan increasing the availability of LARCs in healthcare facilities and ensuring that all citizens are informed about their contraceptive choices.

(18) This aligns with Uganda's goal of achieving universal health coverage for family planning. Although the Uganda Ministry of Health aims to provide LARCs free to all citizens who request one, they acknowledge that availability is often limited due to supply, budget constraints, and trained healthcare providers. $(18,19)$

This study aims to understand the relationship between informed choice and LARC use among women aged 15-49 in Uganda after adjusting for potential confounding. This study will use data from a crosssectional nationally representative sample of women aged 15-49 in Uganda. We hypothesize that women who report informed choice in relation to family planning will have a higher odds of using a LARC method. As such, this study will contribute to a better understanding of family planning counselling in Uganda which may be useful in developing future family planning programs and interventions to promote the usage of LARCs which in turn will help reduce the overall unplanned pregnancy rate in Uganda.

\section{Methods}

\section{Study design and data source}


This study is cross-sectional and utilized data from the 2016 Standard Demographic and Health Survey (DHS) VII for Uganda.(3) The Uganda DHS survey is a nationally representative survey of individuals aged $\geq 15$ years from 112 administrative districts in Uganda. Households were selected using two-stage cluster sampling. In the first stage, 697 enumeration areas were selected which included 162 urban and 535 rural areas. From each enumeration areas, 30 households were randomly selected resulting in a total of 20,910 households. (3) If a household was selected and different occupants lived at the address, the new occupants were interviewed and recorded.(20) This study specifically utilizes data from the Uganda DHS individual dataset, which is a subset of the primary household dataset of surveyed women aged 1549. The overall response rate of the individual dataset was $97 \%(n=18,506)$. Sample weights were included in the dataset. (3)

\section{Study sample}

Women were included in the study if they were (i) aged 15-49; (ii) started a modern form of birth control (i.e., the pill, sterilization, IUD, implant, or injections) within five years preceding the survey; and (iii) responded to at least one informed choice question. Participants with missing values to the exposure or outcome were excluded. In all, 3,768 women met the inclusion criteria for the study (Figure 1).

\section{Study variables}

\section{Outcome}

LARC use is the primary outcome of this study. Women were defined as using a LARC if they indicated that they were currently using an IUD or implant. Women who indicated that they used other forms of modern contraception (i.e., the pill, sterilization, or injectables) were classified as not using a LARC.

\section{Explanatory}

Informed choice is the explanatory variable for this study. The variable was dichotomized into whether or not a woman self-reported informed choice. Informed choice is defined as women who were current users of modern birth control methods who were informed by a healthcare provider about one or more of the following: (i) the side effects or problems of the method used; or (ii) what to do if they experienced side effects or problems with the method used; or (iii) other methods of contraception that could be used.(21)

\section{Potential confounders, predictors, and interactions}

Potential confounders and predictors were based on a literature review and are depicted in the directed acyclic graph (DAG) in Figure 2. Potential demographic predictors included age, $(10,13,22)$ location, $(10$, 23) religion,(10,24) education,(23) economic status, $(22)$ and number of children. $(13,22)$ Other potential predictors included the desire to have more children, $(13,22)$ exposure to family planning messaging, (13) source of birth control, $(10,23)$ and visit to a healthcare facility in the past 12 months.(10) Exposure to family planning messaging is a composite variable of women who indicated that they heard family planning messaging on either the radio, read it in a newspaper, saw it on television, or read a message on 
a mobile phone. Potential confounders include marital status, $(24,25)$ occupation,(10) and ability to access medical care.(13) Finally, based on the literature review, no interaction terms were included in the analysis.

\section{Statistical analysis}

The design-adjusted bivariable analysis was conducted using Thomas-Rao corrections to a chi-square test to test the association between the categorical variables and the outcome.(26) Variance inflated factors (VIF) were used to consider the multicollinearity between variables with a cut off of $\geq 10$. (27) Following, a design-adjusted univariate and multivariable logistic regression was used to build the statistical model to estimate the adjusted effect of informed choice on the use of LARCs.(28) The minimal sufficient adjustment set of confounding variables was determined through a priori knowledge based on the literature review and a constructed DAG.(29) The minimal sufficient adjustment set included age, economic status, education, location, religion, exposure to family planning messaging, and desire for more children. The backward elimination method was used with the additional risk factors determine the final model through the weighted Akaike information criterion (AIC) value. Odds ratios and $95 \%$ confidence intervals $(\mathrm{Cl})$ were provided for the final model. The primary analysis was conducted using complete cases as the number of respondents with missing data was minimal $(<5 \%)$. As such, the data was considered to be missing completely at random. The final model was evaluated using the surveyweighted Receiver Operating Characteristic (ROC) curve statistic(30) and Archer-Lemeshow test.(31)

Propensity score matching was conducted as a sensitivity analysis to assess the robustness of the primary design-adjusted model. The Austin method was used to develop the design-adjusted propensity model in which survey design features were integrated into both the exposure and outcome models. (32) All covariates used in the primary design-adjusted analysis were used in the propensity score logistic model. The nearest neighbor matching without replacement was conducted with a 1:1 match using a caliper of 0.2 to estimate the average treatment effect of the treated. The standard mean difference (SMD) was used to assess the covariate balance with a cut-off of <0.2.(32) After balance was achieved through the SMD, a design-adjusted logistic regression was conducted with the matched data in which LARC use was the outcome and informed choice was the exposure.

Complex sampling design methods, which used sample weights, primary sampling units (PSU), and strata variables, were used in all analyses. The sample weight was divided by 1000000 to normalize it as instructed by the DHS.(21) The PSU "South Buganda Island-urban" was combined with "North Buganda Island-urban" due to low cell counts. All analyses were completed in R 4.0.5.(33) Packages included dplyr, table1, publish, survey, car, MASS, and WeightedROC.

\section{Results}

\section{Demographics}


Descriptive statistics are reported in Table 1. Among the 3,646 women included in the complete case analysis, 975 (26.3\%) reported using a LARC. Descriptive statistics stratified by the outcome are also featured in Table 1. In all, $68.1 \%$ of LARC users reported informed choice compared to $47.9 \%$ of those who did not use a LARC. With regards to women who did not use a LARC, women who reported using a LARC were more likely to be married ( $39.2 \%$ no LARC vs. $44.5 \%$ LARC), obtain their family planning method from a government facility ( $54.4 \%$ no LARC vs, $79.9 \%$ LARC), and be exposed to family planning messaging $(70.4 \%$ no LARC vs. $74.7 \%$ LARC).

\section{Association between LARCs, informed choice, and other potential variables}

In the survey weighted unadjusted model, the odds of reporting LARC use was 2.32 (95\% $\mathrm{Cl} 1.89-2.85$ ) times higher among women who reported informed choice compared to those who did not report informed choice. Table 2 displays the survey-weighted adjusted relationship between informed choice and LARC use. The final design-adjusted multivariable model included age, economic status, education, location, religion, exposure to family planning messaging, desire for more children, source for birth control, and visited a healthcare facility in the past 12 months. In this analysis, the odds of reporting LARC use were $1.92(95 \% \mathrm{Cl} 1.56-2.36)$ times higher among women who reported informed choice compared to those who did not report informed choice.

\section{Propensity score sensitivity analysis}

The total sample size for the propensity score was 3254, with 1627 individuals categorized as informed and 1627 as not informed. All of the included covariates in the propensity score analysis were considered balanced with an SMD of $<0.2$. In the design-adjusted propensity score analysis, the odds of reporting LARC use were 2.37 (95\% $\mathrm{Cl} 1.92$ - 2.94) times higher among women who reported informed choice compared to those who did not report informed choice. (Table 2).

\section{Discussion}

This study included 3646 women using modern contraception in Uganda and, of these, $53.2 \%$ reported that they were informed about their contraceptive method. The results of our analysis demonstrate that women who are informed about family planning in Uganda are approximately twice as likely to choose a LARC birth control method. These results were supported by a sensitivity analysis using propensity score matching in which the findings were similar to the primary analysis in terms of magnitude and direction. As such, these results support our hypothesis that women in Uganda who report informed choice have a higher odds of using a LARC method.

\section{Interpretation}

These results are supported by current literature which notes that women are more likely to choose a LARC over other contraceptive methods when they experience informed choice through access to highquality family planning services and information.(15-17) Although the connection between informed 
choice and LARCs is well established in countries such as the United States(15), there is limited knowledge about informed choice and LARC use in sub-Saharan Africa. A 2020 observational study in Rwanda by Mukamuyango et al. of 1290 couples found that couples that were provided information about LARC and HIV counselling were more likely to use a LARC method. Similarly, within the African continent, Hong et al. published a cross-sectional study in 2006 using DHS data from Egypt that included 8,445 women. They found that women who attended public clinics that offered high-quality family planning services, which included counselling about contraception, were more likely to choose an IUD over other contraceptive methods when compared to individuals who attended public clinics that offered low-quality family planning services (relative risk ratio 1.36; $p$-value $<0.01$ ).(16)

It is also important to discuss the difference in reporting informed choice among women who use a LARC $(68.1 \%)$ versus those who used another modern method (47.9\%) in this study. This difference demonstrates that women are not receiving consistent family planning counselling across different contraceptive methods in Uganda. As discussed at the World Health Organization London Summit on Family Planning in 2012, which was attended by the President of Uganda, all women should have access to comprehensive family planning counselling, regardless of their choice in contraception.(34, 35) However, this finding is unsurprising as access to comprehensive family planning care in Uganda is limited. The Ugandan Ministry of Health acknowledges that many women do not have access to basic health services or providers who are trained in multiple contraceptive methods.(18) In fact, Uganda has one of the lowest numbers of healthcare workers in the world. For example, in 2017 Uganda only had 0.17 physicians per 1000 people while in comparison, the United States had 2.62.(36) This inconsistent availability of healthcare in Uganda is further demonstrated by a 2014 cross-sectional study by Andi et al. of 19,399 women using the 1995-2011 Ugandan DHS data which found significant differences in access to family planning care across Uganda. For example, they found that women living in rural areas in Uganda had 0.89 (95\% $\mathrm{Cl} 0.66$ - 0.99) times the odds of reporting using modern contraception compared to those in urban areas.(37) As such, the findings in this study further highlight the inconsistencies in family planning care provided to women in Uganda.

\section{Strengths and limitations}

The strengths and limitations of this study warrant discussion. This study was strengthened by the use of complex-survey design methods, which allow the findings to be generalizable to the 2016 Ugandan population. As such, the use of population-level data and design-adjusted methods may help reduce selection bias which is common in observational studies.(38) Furthermore, to our knowledge, this is the first study in Uganda that has studied the association between informed choice and LARCs. This is important because the results of this study can be useful for policymakers in Uganda. This study was also strengthened by the propensity sensitivity analysis, which validated the robustness of the primary analysis.

With regards to limitations, the data provided by the DHS is collected through personal interviews and thus subject to recall and social desirability bias which could lead to a lower level of association. 
(38) This may be a concern for the explanatory variable informed choice as women were asked to recall if they were informed about their contraceptive choice. However, this may be partially mitigated by the inclusion of only women who started a modern contraceptive method within the past five years. The study may also be subject to temporal ambiguity bias due to the cross-sectional study design and the relationship between the exposure and outcome.(38) It is possible that some women could identify with informed choice after selecting their method of contraception, which in turn would result in reverse causality.(39) Additionally, the authors recognize that the data is five years old, which could limit its generalizability. However, the findings are consistent with similar studies, which is an indication of its validity. $(15,16)$ Finally, there could be unmeasured and residual confounding in the model created. The authors attempted to mitigate this limitation through the use of a DAG and literature search to better understand how variables interact with one another. However, unmeasured variables, such as the availability of LARCs at the health center, could lower the level of association.

\section{Policy implications and future directions}

As Uganda has one of the highest rates of unplanned pregnancies in the world,(3) there is an urgent need for the Ugandan Ministry of Health to further expand both family planning counselling and the availability of LARCs to achieve coverage goals. This includes training more healthcare providers in LARC methods and expanding access to care. As such, additional family planning funding for LARCs is needed to achieve this goal. Furthermore, additional research is needed on the type of counselling that is currently being provided to women in Uganda. Research should consider both providers and contraceptive users. By better understanding how women are currently being counselled and what contraceptive method they are choosing, policymakers and stakeholders can further develop comprehensive family planning programs in not only Uganda but similar countries as well.

\section{Conclusion}

This nationally representative study found that among women aged 15-49 in Uganda, those who reported informed choice in relation to family planning were approximately twice as likely to report using a LARC compared to those who used another modern contraceptive method. As such, the Ugandan Ministry of Health should further expand access to family planning counselling as it could contribute to the reduction of unplanned pregnancies across Uganda through the use of LARCs.

\section{References}

1. Winner B, Peipert JF, Zhao Q, et al. Effectiveness of Long-Acting Reversible Contraception. New England Journal of Medicine. 2012; 366(21):1998-2007. doi:10.1056/NEJMoa1110855

2. Stoddard A, McNicholas C, Peipert JF. Efficacy and Safety of Long-Acting Reversible Contraception. Drugs. 2011; 71(8):969-80. doi:10.2165/11591290-000000000-00000 
3. Uganda Bureau of Statistics (UBOS) and ICF. Uganda Demographic and Health Survey 2016. Kampala, Uganda and Rockville, Maryland, USA: UBOS and ICF2018.

4. United Nations Department of Economic and Social Affairs Population Division. Contraceptive Use by Method 2019: Data Booklet United Nations, Department of Economic and Social Affairs New York; 2019.

5. Adedini SA, Omisakin OA, Somefun OD. Trends, patterns and determinants of long-acting reversible methods of contraception among women in sub-Saharan Africa. PloS one. 2019; 14(6):e0217574-e. doi:10.1371/journal.pone.0217574

6. Valdés PR, Alarcon AM, Munoz SR. Evaluation of Informed Choice for contraceptive methods among women attending a family planning program: conceptual development; a case study in Chile. Journal of Clinical Epidemiology. 2013; 66(3):302-7. doi:https://doi.org/10.1016/j.jclinepi.2012.07.003

7. Tumlinson K, Pence BW, Curtis SL, et al. Quality of Care and Contraceptive Use in Urban Kenya. Int Perspect Sex Reprod Health. 2015; 41(2):69-79. doi:10.1363/4106915

8. Fruhauf T, Zimmerman L, Kibira SPS, et al. Measuring family planning quality and its link with contraceptive use in public facilities in Burkina Faso, Ethiopia, Kenya and Uganda. Health Policy and Planning. 2018; 33(7):828-39. doi:10.1093/heapol/czy058

9. Tran NT, Seuc A, Coulibaly A, et al. Post-partum family planning in Burkina Faso (Yam Daabo): a two group, multi-intervention, single-blinded, cluster-randomised controlled trial. The Lancet Global Health. 2019; 7(8):e1109-e17. doi:https://doi.org/10.1016/S2214-109X(19)30202-5

10. Handebo S. Informed Choice of Contraceptive Methods among Women in Ethiopia: Further Analysis of the 2016 Ethiopian Demographic Health Survey. Open Access Journal of Contraception. 2021; 12:83.

11. Anguzu R, Tweheyo R, Sekandi JN, et al. Knowledge and attitudes towards use of long acting reversible contraceptives among women of reproductive age in Lubaga division, Kampala district, Uganda. BMC Research Notes. 2014; 7(1):153. doi:10.1186/1756-0500-7-153

12. Kim YM, Kols A, Mucheke S. Informed Choice and Decision-Making in Family Planning Counseling in Kenya. International Family Planning Perspectives. 1998; 24(1):4-42. doi:10.2307/2991913

13. Wado YD, Gurmu E, Tilahun T, et al. Contextual influences on the choice of long-acting reversible and permanent contraception in Ethiopia: a multilevel analysis. PloS one. 2019; 14(1):e0209602.

14. Hussain R. Unintended pregnancy and abortion in Uganda. Washington DC: Guttmacher Institute2013. 
15. Secura GM, Allsworth JE, Madden T, et al. The Contraceptive CHOICE Project: reducing barriers to long-acting reversible contraception. American Journal of Obstetrics and Gynecology. 2010; 203(2):115.e1-.e7. doi:https://doi.org/10.1016/j.ajog.2010.04.017

16. Hong R, Montana L, Mishra V. Family planning services quality as a determinant of use of IUD in Egypt. BMC Health Services Research. 2006; 6(1):79. doi:10.1186/1472-6963-6-79

17. Mukamuyango J, Ingabire R, Parker R, et al. Uptake of long acting reversible contraception following integrated couples HIV and fertility goal-based family planning counselling in Catholic and nonCatholic, urban and rural government health centers in Kigali, Rwanda. Reproductive Health. 2020; 17(1):126. doi:10.1186/s12978-020-00981-0

18. Republic of Uganda Ministry of Health. National Family Planning Advocacy Strategy Costed Implementation Plan 2020 - 2025. Uganda2020.

19. Republic of Uganda Ministry of Health. Annual Health Sector Performance Report. Kampala, Uganda2020.

20. ICF International. Demographic and Health Survey Interviewer's Manual. Calverton, Maryland2012.

21. Croft TN, Marshall AM, Allen CK, et al. Guide to DHS statistics. Rockville: ICF. 2018; 645.

22. Bhandari R, Pokhrel KN, Gabrielle N, et al. Long acting reversible contraception use and associated factors among married women of reproductive age in Nepal. PLOS ONE. 2019; 14(3):e0214590. doi:10.1371/journal.pone.0214590

23. Jadhav A, Vala-Haynes E. Informed choice and female sterilization in South Asia and Latin America. Journal of biosocial science. 2018; 50(6):823-39. doi:10.1017/S0021932017000621

24. Ahinkorah BO. Predictors of modern contraceptive use among adolescent girls and young women in sub-Saharan Africa: a mixed effects multilevel analysis of data from 29 demographic and health surveys. Contraception and Reproductive Medicine. 2020; 5(1):32. doi:10.1186/s40834-020-00138-1

25. Kungu W, Khasakhala A, Agwanda A. Use of long-acting reversible contraception among adolescents and young women in Kenya. PLOS ONE. 2020; 15(11):e0241506.

doi:10.1371/journal.pone.0241506

26. Rao JN, Scott AJ. On chi-squared tests for multiway contingency tables with cell proportions estimated from survey data. The Annals of statistics. 1984:46-60.

27. Leeuwenberg AM, van Smeden M, Langendijk JA, et al. Performance of binary prediction models in high-correlation low-dimensional settings: a comparison of methods. Diagnostic and Prognostic Research. 2022; 6(1):1. doi:10.1186/s41512-021-00115-5 
28. Koch GG, Freeman Jr DH, Freeman JL. Strategies in the multivariate analysis of data from complex surveys. International Statistical Review/Revue Internationale de Statistique. 1975:59-78.

29. Knüppel S, Stang A. DAG program: identifying minimal sufficient adjustment sets. Epidemiology (Cambridge, Mass.). 2010; 21(1):159. doi:10.1097/EDE.0b013e3181c307ce

30. Yao W, Li Z, Graubard BI. Estimation of ROC curve with complex survey data. Statistics in medicine. 2015; 34(8):1293-303.

31. Archer KJ, Lemeshow S. Goodness-of-fit test for a logistic regression model fitted using survey sample data. The Stata Journal. 2006; 6(1):97-105.

32. Austin PC. An Introduction to Propensity Score Methods for Reducing the Effects of Confounding in Observational Studies. Multivariate Behav Res. 2011; 46(3):399-424. doi:10.1080/00273171.2011.568786

33. R Core Team. R: A language and environment for statistical computing. Vienna, Austria: R Foundation for Statistical Computing; 2021.

34. World Health Organization, editor. Summit on Family Planning; 2012; London, United Kingdom.

35. Cohen SA. London Summit Puts Family Planning Back on the Agenda, Offers New Lease on Life for Millions of Women and Girls. Guttmacher Policy Review. 2012; 15(3).

36. The World Bank. Physicians (per 1,000 people). Washington DC.

2021. https://data.worldbank.org/indicator/SH.MED.PHYS.ZS. Accessed December 62021.

37. Andi JR, Wamala R, Ocaya B, et al. Modern contraceptive use among women in Uganda: An analysis of trend and patterns (1995-2011). Etude Popul Afr. 2014; 28(2):1009-21. doi:10.11564/28-0-553

38. Delgado-Rodriguez M, Llorca J. Bias. Journal of Epidemiology \& Community Health. 2004; 58(8):635-41.

39. Kramer MS. Cross-sectional studies. Clinical epidemiology and biostatistics: Springer; 1988. p. 113-7.

\section{Tables}

Table 1. Design-adjusted characteristics of Ugandan women aged 15-49 using modern birth control stratified by use of long-acting reversible contraception: 2016 Uganda Demographic and Health Survey data 
Variable name

No LARC LARC $^{\mathrm{a}} \quad$ Overall P-value ${ }^{\mathrm{b}, \mathrm{c}}$

\begin{tabular}{lllll} 
& $\mathrm{n}\left(\%^{\mathrm{b}}\right)$ & $\mathrm{n}\left(\%^{\mathrm{b}}\right)$ & $\mathrm{n}\left(\%^{\mathrm{b}}\right)$ & \\
\hline Sample Size & $2671(73.7)$ & $975(26.3)$ & $3646(100.0)$ & \\
\hline Informed choice & & & & \\
\hline No & $1373(52.1)$ & $300(31.9)$ & $1973(46.8)$ & $<0.001$ \\
\hline Yes & $1298(47.9)$ & $675(68.1)$ & $1673(53.2)$ & \\
\hline Age & & & & 0.01 \\
\hline $15-18$ & $190(7.5)$ & $43(4.6)$ & $233(6.7)$ & \\
\hline $19-29$ & $1269(48.1)$ & $427(45.3)$ & $1696(47.3)$ & \\
\hline $30-39$ & $898(32.8)$ & $371(36.9)$ & $1269(33.9)$ & \\
\hline $40-49$ & $314(11.6)$ & $134(13.2)$ & $448(12.0)$ & \\
\hline Location & & & & \\
\hline Urban & $614(26.4)$ & $253(28.8)$ & $867(27.0)$ & 0.275 \\
\hline Rural & $2057(73.6)$ & $722(71.2)$ & $2779(73.0)$ & \\
\hline Religion & & & & \\
\hline Anglican & $896(32.6)$ & $372(38.3)$ & $1268(34.1)$ & 0.016 \\
\hline Catholic & $1033(38.2)$ & $383(37.6)$ & $1416(38.0)$ & \\
\hline Muslim & $355(14.5)$ & $85(10.7)$ & $440(13.5)$ & \\
\hline Other & $387(14.7)$ & $135(13.5)$ & $522(14.4)$ & \\
\hline Education & & & & \\
\hline & & & & \\
\hline
\end{tabular}

Education

\begin{tabular}{lllll}
\hline No Education & $215(7.7)$ & $66(6.2)$ & $281(7.3)$ & 0.001 \\
\hline Primary & $1639(58.3)$ & $613(61.4)$ & $2252(59.1)$ & \\
\hline Secondary & $651(27.1)$ & $203(21.7)$ & $854(25.7)$ \\
\hline Higher & $166(6.9)$ & $93(10.7)$ & $259(7.9)$ \\
\hline
\end{tabular}

Marital status

\begin{tabular}{lllll}
\hline Never married & $170(6.6)$ & $58(6.3)$ & $228(6.6)$ & 0.005 \\
\hline Married & $1100(39.2)$ & $440(44.5)$ & $1540(40.6)$ \\
\hline Cohabiting & $1089(41.5)$ & $331(34.1)$ & $1420(39.6)$ \\
\hline Widowed/divorced/separated & $312(12.7)$ & $146(15.0)$ & $458(13.3)$ \\
\hline
\end{tabular}


Occupation

\begin{tabular}{lllll} 
Unemployed & $397(15.8)$ & $122(13.9)$ & $519(15.3)$ & 0.456 \\
\hline Agriculture & $1153(40.7)$ & $415(42.3)$ & $1568(41.2)$ \\
\hline Non-Agriculture & $1121(43.4)$ & $438(43.9)$ & $1559(43.5)$
\end{tabular}

${ }^{a}$ Long-acting reversible contraception

${ }^{\mathrm{b}}$ Weighted estimates

${ }^{\mathrm{c}}$ P-values estimated using the Thomas-Rao modification chi-square 
Variable name

$\begin{array}{llll}\text { No LARC } & \text { LARC } & \text { Overall } & \text { P- } \\ \mathrm{n}\left(\%^{\mathrm{b}}\right) & \mathrm{n}\left(\%^{\mathrm{b}}\right) & \mathrm{n}\left(\%^{\mathrm{b}}\right) & \text { value }^{\mathrm{b}, \mathrm{c}}\end{array}$

Economic status

\begin{tabular}{|c|c|c|c|}
\hline Lowest & $399(12.7)$ & $\begin{array}{l}158 \\
(14.6)\end{array}$ & $557(13.2)$ \\
\hline Second & $556(18.8)$ & $\begin{array}{l}198 \\
(19.2)\end{array}$ & 754 (18.9) \\
\hline Middle & 578 (20.7) & $\begin{array}{l}181 \\
(19.3)\end{array}$ & 759 (20.3) \\
\hline Fourth & $564(22.5)$ & $\begin{array}{l}217 \\
(21.6)\end{array}$ & 781 (22.3) \\
\hline Highest & $574(25.3)$ & $\begin{array}{l}221 \\
(25.3)\end{array}$ & 795 (25.3) \\
\hline
\end{tabular}

Number of children

$\begin{array}{lllll}\text { None } & 97(3.7) & 11(1.2) & 108(3.1) & <0.001 \\ 1-2 & 905(35.1) & 294 & \begin{array}{l}1199 \\ (30.9)\end{array} & (34.0)\end{array}$

\section{3-4}

$\begin{array}{lll}815(30.7) & 335 & 1150 \\ & (35.0) & (31.9)\end{array}$

$5+$

854 (30.5) $\quad 335$

(32.9) (31.1)

Want more children

\begin{tabular}{lllll} 
No & $\begin{array}{l}1076 \\
(39.7)\end{array}$ & $\begin{array}{l}428 \\
(43.3)\end{array}$ & $\begin{array}{l}1504 \\
(40.6)\end{array}$ & 0.173 \\
\hline Yes & 1527 & 520 & 2047 & \\
& $(58.0)$ & $(54.1)$ & $(57.0)$ & \\
\hline Undecided & $68(2.4)$ & $27(2.6)$ & $95(2.4)$ & \\
\hline Exposure to family planning messaging & & & & \\
\hline No & $809(29.6)$ & 251 & 1060 & 0.031 \\
& & $(25.3)$ & $(28.4)$ & \\
Yes & 1862 & 724 & 2586 & \\
& $(70.4)$ & $(74.7)$ & $(71.6)$ & \\
Source for birth control & & & & \\
Government & 1523 & 788 & 2311 & \\
& $(54.4)$ & $(79.9)$ & $(61.1)$ & \\
\hline Private & 1002 & 181 & 1183 & \\
& Page $14 / 18$ & & & \\
\hline
\end{tabular}


Visited a healthcare facility in the past 12 months

\begin{tabular}{|c|c|c|c|c|}
\hline No & $681(25.4)$ & $\begin{array}{l}247 \\
(25.6)\end{array}$ & $928(25.4)$ & 0.002 \\
\hline Yes & $\begin{array}{l}1990 \\
(74.6)\end{array}$ & $\begin{array}{l}728 \\
(74.4)\end{array}$ & $\begin{array}{l}2718 \\
(74.6)\end{array}$ & \\
\hline \multicolumn{5}{|c|}{ Ability to access medical care } \\
\hline Big problem & $\begin{array}{l}1009 \\
(35.9)\end{array}$ & $\begin{array}{l}354 \\
(35.5)\end{array}$ & $\begin{array}{l}1363 \\
(35.8)\end{array}$ & 0.426 \\
\hline Not a big problem & $\begin{array}{l}1662 \\
(64.1)\end{array}$ & $\begin{array}{l}621 \\
(64.5)\end{array}$ & $\begin{array}{l}2283 \\
(64.2)\end{array}$ & \\
\hline
\end{tabular}

Table 1 continued. Design-adjusted characteristics of Ugandan women aged 15-49 using modern birth control stratified by use of long-acting reversible contraception: 2016 Uganda Demographic and Health Survey data

${ }^{a}$ Long-acting reversible contraception

${ }^{\mathrm{b}}$ Weighted estimates

${ }^{c}$ P-values estimated using the Thomas-Rao modification chi-square

Table 2. Design-adjusted estimates from the multivariable logistic regression and propensity score sensitivity analysis assessing the relationship between informed choice and LARC use among Ugandan women aged 15-49: 2016 Uganda Demographic and Health Survey data 
$\mathrm{aOR}^{\mathrm{a}}\left(95 \% \mathrm{Cl}^{\mathrm{b}}\right)$

\section{Multivariable logistic regression}

\begin{tabular}{ll} 
LARC $^{C}$ use & \\
\hline No & Reference \\
\hline Yes & $1.98(1.61,2.43)^{d, e, f}$
\end{tabular}

\section{Propensity score sensitivity analysis}

\begin{tabular}{ll}
$\mathrm{LARC}^{C}$ use & \\
\hline No & Reference \\
\hline Yes & $2.37(1.92-2.94)^{\mathrm{g}}$
\end{tabular}

${ }^{\text {a }}$ Adjusted odds ratio

b $95 \%$ confidence interval

${ }^{c}$ Long-acting reversible contraception

d Adjusted for age, economic status, education, location, religion, exposure to family planning messaging, desire for more children, source for birth control, and visited a healthcare facility in the past 12 months

${ }^{\text {e }}$ Area under the curve (AUC) $=0.71$, which indicates a fair ability to discriminate.(42)

${ }^{\mathrm{f}}$ Archer-Lemeshow test $=.33$, which indicates that there is not a lack of fit.(31)

g Propensity score was calculated using predictors: age, religion, location, economic status, education, want more children, and exposure to family planning messaging

\section{Figures}




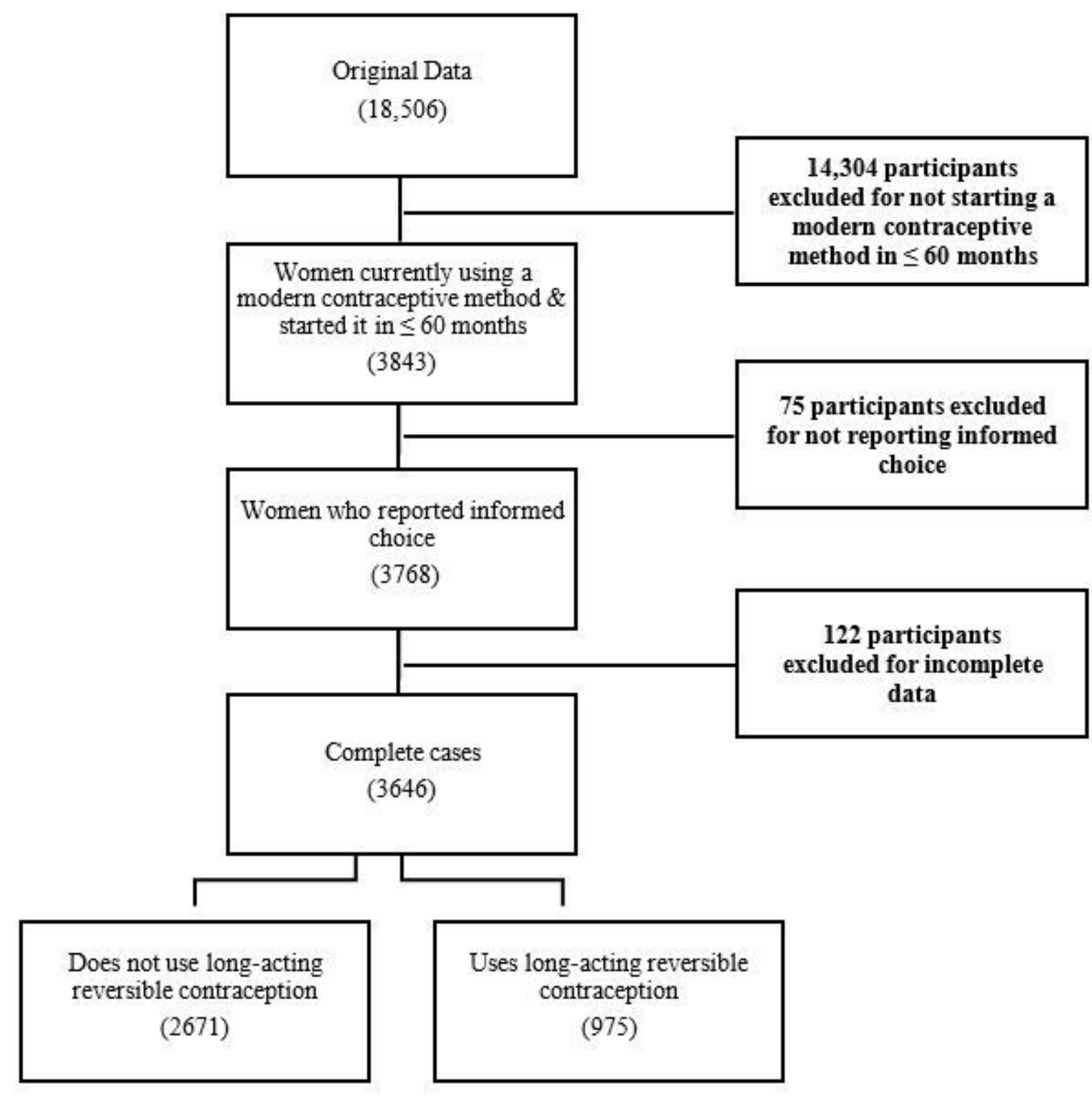

\section{Figure 1}

Flow chart depicting the sample selection of women aged 15-49 in Uganda using modern contraception and responding to informed choice questions from the women's dataset using the 2016 Uganda Demographic and Health Survey. 


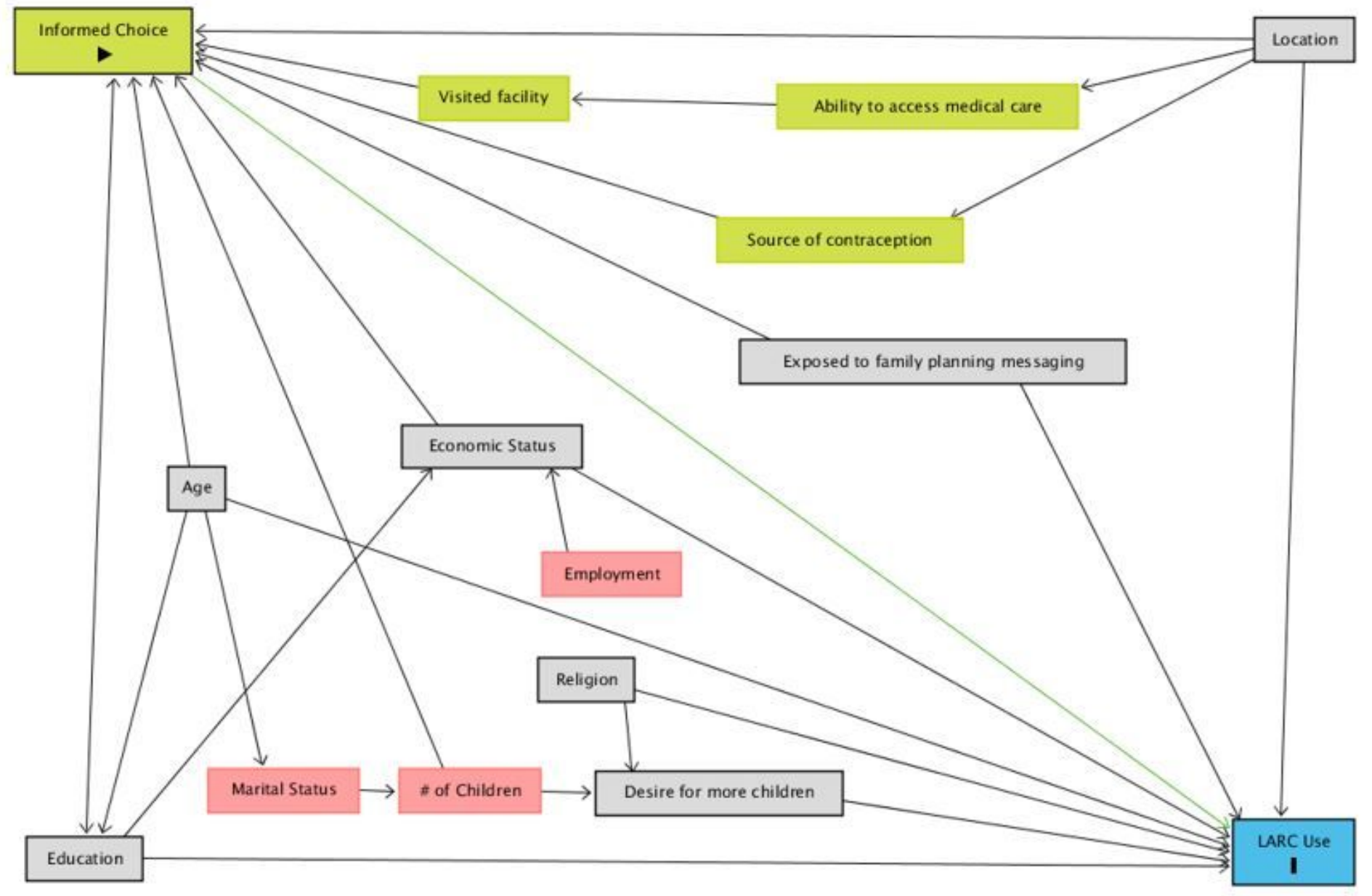

Figure 2

Directed acyclic graph (DAG) depicting the adjusted effect of informed choice on long-acting reversible contraception (LARC) use. The following variables have been adjusted as part of the minimal sufficient adjustment set: education, age, religion, economic status, location, desire for more children, and exposed to family planning messaging 